\title{
Ab initio no core full configuration approach for light nuclei
}

\author{
Youngman Kim* and Ik Jae Shin \\ Rare Isotope Science Project, Institute for Basic Science, Daejeon 305-811, Korea \\ *E-mail:ykim@ibs.re.kr,geniean@ibs.re.kr \\ Pieter Maris and James P. Vary \\ Department of Physics and Astronomy, Iowa State University, Ames, IA 50011, USA \\ E-mail:pmaris@iastate.edu, jvary@iastate.edu \\ Christian Forssén and Jimmy Rotureau \\ Department of Fundamental Physics, Chalmers University of Technology, SE-412 96 \\ Göteborg, Sweden \\ E-mail: christian.forssen@chalmers.se,rotureau@chalmers.se
}

\begin{abstract}
Comprehensive understanding of the structure and reactions of light nuclei poses theoretical and computational challenges. Still, a number of ab initio approaches have been developed to calculate the properties of atomic nuclei using fundamental interactions among nucleons. Among them, we work with the ab initio no core full configuration (NCFC) method and ab initio no core Gamow Shell Model (GSM). We first review these approaches and present some recent results.
\end{abstract}

Keywords: nuclear structure; ab initio approach.

\section{Introduction}

Nuclei are complicated quantum many-body systems and offer a solid testing ground for our knowledge of the strong interaction in the nonperturbative regime. It is a formidable task to get a firm grasp of how stable (and unstable) nuclei emerge from protons and neutrons whose interactions are dominated by the strong interaction. With the rapid growth of available high performance supercomputers, several ab initio approaches have been developed to study nuclear structures and reactions based on fundamental nuclear interactions. Robust and reliable results from ab initio methods may provide a clue to the role of fundamental degrees of freedom such as quarks in nuclei. 
In this work, we study the properties of ${ }^{6} \mathrm{Li}$ using the ab initio no core full configuration (NCFC) ${ }^{1}$ and no core Gamow Shell Model (GSM) ${ }^{2}$ approaches with two different nucleon-nucleon $(\mathrm{NN})$ interactions; the inversescattering interaction JISP16 and the new $\mathrm{NNLO}_{\text {opt }}$ potential ${ }^{3}$ from chiral effective field theory (chEFT). We first review these approaches and present some of our recent results. ${ }^{4}$

\section{Ab initio no core full configuration approach}

We start with the configuration interaction (CI) method on which the ab initio no core full configuration (NCFC) method is based. In short, the CI method is one of the post Hartree-Fock methods for solving the Schrödinger equation using a matrix formulation. The A-body Schrödinger equation is

$$
\hat{H} \Psi\left(\mathbf{r}_{1}, \mathbf{r}_{2}, \ldots, \mathbf{r}_{A}\right)=E \Psi\left(\mathbf{r}_{1}, \mathbf{r}_{2}, \ldots, \mathbf{r}_{A}\right),
$$

where the Hamiltonian $\hat{H}$ contains kinetic energy and interaction terms. In contrast to the Hartree-Fock method, where the A-body wave function is approximated by a single Slater determinant, the A-body wave function in the CI method is given by a linear combination of Slater determinants $\Phi_{i}$ :

$$
\Psi\left(\mathbf{r}_{1}, \mathbf{r}_{2}, \ldots, \mathbf{r}_{A}\right)=\sum_{i=0}^{k} c_{i} \Phi_{i}\left(\mathbf{r}_{1}, \mathbf{r}_{2}, \ldots, \mathbf{r}_{A}\right) .
$$

To obtain the exact A-body wave function one has to consider infinite number of configurations, $k=\infty$, in practice, however, the sum must be limited to a finite number of configurations. The Slater determinant is the antisymmetrized product of single particle wave functions $\phi_{\alpha}(\mathbf{r})$, where $\alpha$ denotes the quantum numbers of single particle states. A traditional choice for the single particle basis is that of harmonic oscillator. Now, the matrix elements of the Hamiltonian is given by $H_{i j}=\left\langle\Phi_{i}|\hat{H}| \Phi_{j}\right\rangle$. For large and sparse matrices, the Lanczos method ${ }^{5}$ has been widely used to find the extreme eigenvalues. This method is implemented in MFDn, ${ }^{6-8}$ a hybrid MPI/OpenMP CI code for ab initio nuclear structure calculations.

Now we move on to the NCFC approach. This method is a version of the ab initio no core shell model (NCSM) with a few important characteristics that will be outlined below. The NCSM treats all nucleons in a nucleus as active and dynamical degrees of freedom. There is no postulated closed, inert core of nucleons in the nucleus. In the ab initio NCSM we start with the intrinsic Hamiltonian of A nucleons

$$
H_{A}=\frac{1}{A} \sum_{i<j} \frac{\left(\mathbf{p}_{i}-\mathbf{p}_{j}\right)^{2}}{2 m}+\sum_{i<j} V_{\mathrm{NN}, i j}+\sum_{i<j<k} V_{\mathrm{NNN}, i j k}
$$


and add the harmonic oscillator (HO) center of mass Hamiltonian. Here, $m$ is the nucleon mass, and $V_{\mathrm{NN}}\left(V_{\mathrm{NNN}}\right)$ is a two-nucleon (three-nucleon) interaction. In the NCSM, the HO basis is employed. Due to the strong short-range correlations of nucleons in a nucleus, a large model space is required to achieve convergence. This infinite (or very large) model space problem might be overcome by the use of effective interactions rather than bare ones. For more on the NCSM, we refer to a recent review article. ${ }^{9}$

Features of the NCFC approach are; (1) the use of interactions defined for an infinite Hilbert space, (2) extrapolating to the continuum limit (infinite matrix limit), and (3) uncertainty estimation for the extrapolation.

Next, we discuss the interactions adopted in the current work. In the present study, we are using the JISP16 phenomenological and $\mathrm{NNLO}_{\text {opt }}$ chiral NN potentials. JISP (J-matrix Inverse Scattering Potential) type interactions ${ }^{10-12}$ are constructed in the framework of the J-matrix version of inverse scattering theory. The matrix elements of the NN potential are calculated in the oscillator basis for each partial wave to reproduce experimental NN scattering data and deuteron properties without three-nucleon interactions. The JISP16 potential is obtained to fit the experimental data for light nuclei up to $A=16$. A promising approach to construct and understand the nuclear force from first principles is chEFT. ${ }^{13}$ An important and up-to-date optimization of the chiral Next-to-Next-to-Leading Order (NNLO) potential was performed using POUNDERS (Practical Optimization Using No Derivatives for Squares), to obtain the potential we label as $\mathrm{NNLO}_{\text {opt }} \cdot{ }^{3}$ The new chiral $\mathrm{NNLO}_{\text {opt }}$ yields $\chi^{2} \approx 1$ per degree of freedom for laboratory energies roughly less than $125 \mathrm{MeV}$. It is also observed that the effects of three-nucleon interactions on the properties of light nuclei with $A=3,4$ are smaller than previously available parameterizations of chiral nuclear forces.

We now address the extrapolation to infinite matrix limit. We work with the $N_{\max }$ truncation scheme, where $N_{\max }$ is the basis truncation parameter. In this scheme, we consider all possible configurations with $N_{\max }$ excitations above the unperturbed ground state: $\sum N_{i} \leq N_{0}+N_{\max }$. Here, $N_{0}$ is the total number of $\mathrm{HO}$ quanta for the ground state configuration and $N_{i}$ is the number of quanta for each state. To take the infinite matrix limit, several extrapolation methods have been developed. ${ }^{1,14-17}$

Finally, we show a few results from our NCFC study. ${ }^{4}$ In Fig. 1, we show the convergence of the ground state energy of ${ }^{6} \mathrm{Li}$ with the extrapolation $\mathrm{A},{ }^{1}$ while excitation energies are presented in Fig. 2. The results are obtained from computations in model spaces up to $N_{\max }=16$ (matrix dimension 
$\left.8 \times 10^{8}\right)$. For a previous study on the ${ }^{6} \mathrm{Li}$ in the ab initio NCFC method, we refer to the work by Cockrell, et al, ${ }^{18}$ where lithium isotopes, ${ }^{6} \mathrm{Li},{ }^{7} \mathrm{Li}$, and ${ }^{8} \mathrm{Li}$, are studied with the JISP16 interaction.

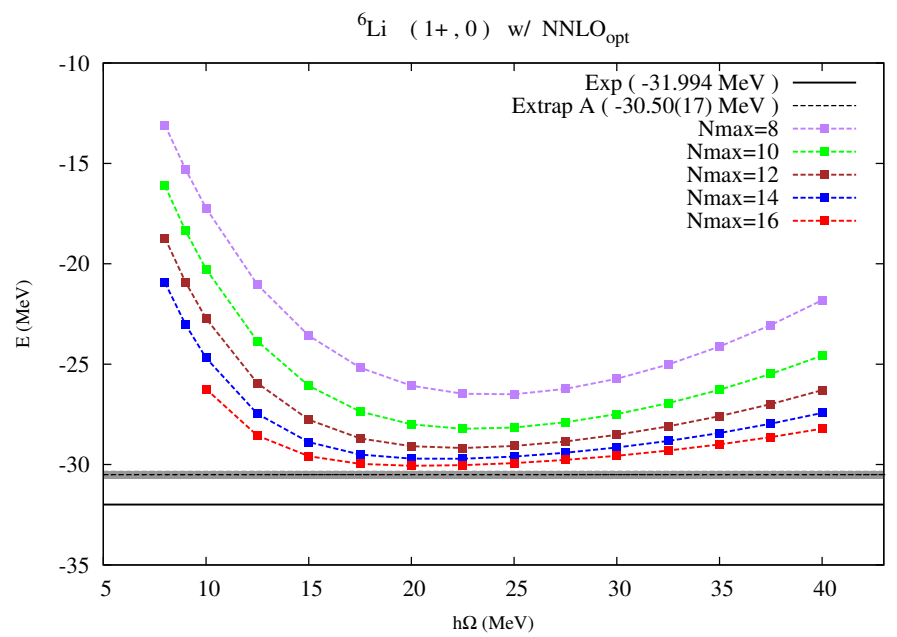

Fig. 1. Ground state energy of ${ }^{6} \mathrm{Li}$ calculated with $\mathrm{NNLO}_{\text {opt }}$ as a function of the size of HO basis $N_{\max }$ and the result with the extrapolation A. The shaded area around the extrapolation A result indicates our estimated uncertainty of $170 \mathrm{keV}$.

\section{The ab initio Gamow Shell Model}

As one approaches the particle emission thresholds, it becomes increasingly important to describe correctly the coupling to the continuum of decays and scattering channels. The recently developed complex-energy Gamow Shell Model (GSM $)^{19}$ has proven to be a reliable tool in the description of nuclei, where continuum effects cannot be neglected. In the GSM, the many-body basis is constructed from a single-particle Berggren ensemble ${ }^{20}$ which includes bound, resonant and complex-continuum states. For practical calculations, the set of continuum states is discretized. As in any Shell Model calculation the dimension of the Hamiltonian matrix grows rapidly with the number of single-particle states and the number of nucleons. In addition, the Hamiltonian matrix in our rigged Hilbert space is non-Hermitian (complex symmetric). Hence, advanced numerical methods that can handle large non-Hermitian matrices must be used. In the context of the GSM, it has been shown that the Density Matrix Renormalization Group (DMRG) 


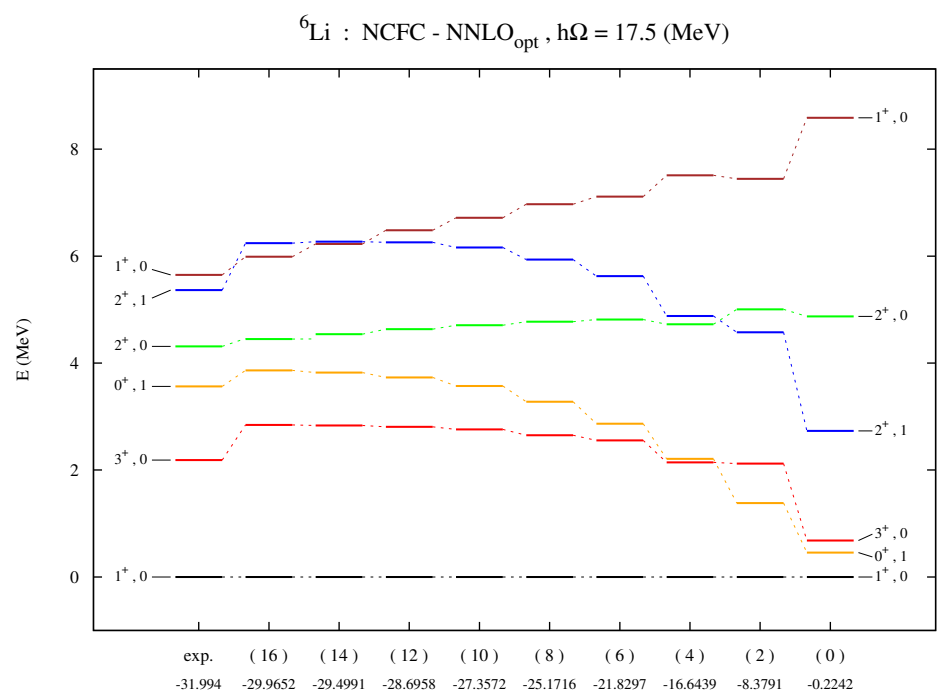

Fig. 2. Excitation energies of ${ }^{6} \mathrm{Li}$ calculated with $\mathrm{NNLO}_{\text {opt }}$ and experimental data. The low-lying positive parity states are shown as a function of $N_{\max }$ truncation (indicated in parenthesis below each column). The ground state eigenvalue (in $\mathrm{MeV}$ ) is also listed for each $N_{\max }$.

is an efficient way to compute the low-lying spectrum of the Hamiltonian at a low computational cost. ${ }^{21}$

Let us consider the application of the $J$-scheme DMRG in the context of the GSM (GSM+DMRG). The objective is to calculate an eigenstate $\left|J^{\pi}\right\rangle$ of the GSM Hamiltonian $\hat{H}$ with angular momentum $J$ and parity $\pi$. As $\left|J^{\pi}\right\rangle$ is a many-body pole of the scattering matrix of $\hat{H}$, the contribution from non-resonant scattering shells along the continuum contour $L^{+}$to the many-body wave function is usually smaller than the contribution from the resonant orbits. ${ }^{19}$ Based on this observation, the following separation is usually performed: ${ }^{21}$ the many-body states constructed from the singleparticle poles form a subspace $A$ (the so-called 'reference subspace'), and the remaining states containing contributions from non-resonant shells form a complement subspace $B$.

One begins by constructing states $|k\rangle_{A}$ forming the reference subspace A. All possible matrix elements of suboperators of the GSM Hamiltonian $\hat{H}$ acting in $A$, expressed in the second quantization form, are then calculated and stored and the GSM Hamiltonian is diagonalized in the reference space to provide the zeroth-order approximation $\left|\Psi_{J}\right\rangle^{(0)}$ to $\left|J^{\pi}\right\rangle$. This vector, called 'reference state', plays an important role in the GSM+DMRG 
truncation algorithm. The scattering shells $(l j)$, belonging to the discretized contour $L^{+}$, are then gradually added to the reference subspace to create the subspace $B$. This first stage of the GSM+DMRG procedure is referred to as the warm-up phase. For each new shell that is added, all possible many-body states denoted as $|i\rangle_{B}$ are constructed and matrix elements of suboperators of the GSM Hamiltonian acting on $|i\rangle_{B}$ are computed. By coupling states in $A$ with the states $|i\rangle_{B}$, one constructs the set of states of a given $J^{\pi}$. This ensemble serves as a basis in which the GSM Hamiltonian is diagonalized. The target state $\left|\Psi_{J}\right\rangle$ is selected among the eigenstates of $\hat{H}$ as the one having the largest overlap with the reference vector $\left|\Psi_{J}\right\rangle^{(0)}$. Then, the desired truncation is performed in $B$ by introducing the reduced density matrix, constructed by summing over the reference subspace $A$. The GSM density matrix being complex-symmetric, the truncation is done by keeping the eigenstates $\alpha_{B}$ (the 'optimized' states) with the largest nonzero moduli of eigenvalue $w_{\alpha} \cdot{ }^{21}$

The warm-up phase is followed by the so-called sweeping phase, in which, starting from the last scattering shell $(l j)_{\text {last }}$, the procedure continues in the reverse direction (the 'sweep-down' phase) until the first scattering shell is reached. The procedure is then reversed and a sweep in the upward direction (the 'sweep up' phase) begins. The sweeping sequences continue until convergence for target eigenvalue is achieved.

A no core GSM+DMRG approach was recently developed ${ }^{2}$ to be used for ab initio studies of light nuclei using realistic interactions. Here we show an application of the DMRG method for the $J^{\pi}=1^{+}$ground state in ${ }^{6} \mathrm{Li}$. Since this state is well bound, the effects of the coupling to the continuum states are negligible. Nevertheless, for the purpose of illustration, we show results using the DMRG technique in a model space containing only HO shells. The model space includes proton and neutron shells with energy up to $10 \hbar \omega$ that is, we include s-shells up to the $5 s_{1 / 2}$, p-shells up to $4 p_{1 / 2 ; 3 / 2}$ and d-shells up to $4 d_{3 / 2 ; 5 / 2}$. For this calculation which serves as an illustration of the method, we are not including shells with higher lvalues. In Fig. 3 we show results obtained by keeping the eigenstates of the density matrix such that $\epsilon=1-\sum_{\alpha} w_{\alpha} \leq 5 \times 10^{-6}$. Results are shown starting from the middle of the warm-up phase until the end of the second sweep. The relatively small difference between the lowest and highest energy during the second sweep $(\sim 360 \mathrm{keV})$ could be further decreased by keeping more states. ${ }^{21}$ The dimension of the total model space in the $J$-scheme is $141,762,900$ whereas the largest DMRG matrix to be diagonalized has a dimension equal to 68,386 . 


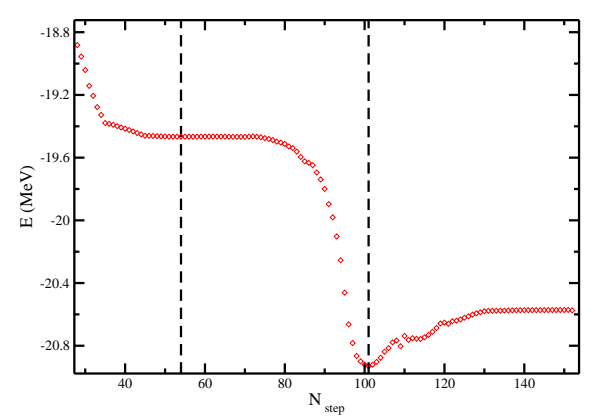

Fig. 3. Iterative process of the DMRG approach for $\epsilon=5 \times 10^{-6}$ and including only waves up to $l=2(\mathrm{~s}, \mathrm{p}, \mathrm{d})$. Results are shown starting from the middle of the warm-up phase, and the two vertical dashed lines show respectively the beginning of the first and second sweeping phase.

\section{Summary}

We briefly introduced the ab initio NCSM, NCFC method, and ab initio GSM approach. To study the properties of ${ }^{6} \mathrm{Li}$, we employed the JISP16 realistic nucleon-nucleon potential and chiral $\mathrm{NNLO}_{\text {opt }}$ interaction. We showed some of our recent results in Figs. 1, 2, 3. From Figs. 1, 2, we conclude that sufficient convergence is achieved in our study.

\section{Acknowledgments}

The work of YK and IJShin was supported by the Rare Isotope Science Project of Institute for Basic Science funded by Ministry of Science, ICT and Future Planning and National Research Foundation of Korea (2013M7A1A1075766). This work was supported in part by the U.S. National Science Foundation under Grant No. PHY-0904782 and the U.S. Department of Energy under Grant Nos. DE-FG02-87ER40371 and DESC0008485 (SciDAC-3/NUCLEI). The research leading to these results has received funding from the European Research Council under the European Community's Seventh Framework Programme (FP7/2007-2013) / ERC grant agreement no. 240603. A portion of the computational resources were provided by the National Energy Research Scientific Computing Center (NERSC), which is supported by the U.S. DOE Office of Science under Contract No. DE-AC02-05CH11231. Computational resources were also provided by the Supercomputing Center/Korea Institute of Science and Technology Information including technical support (KSC-2012-C3-054). 


\section{References}

1. P. Maris, J. P. Vary and A. M. Shirokov, Phys. Rev. C 79 (2009) 014308.

2. G. Papadimitriou, J. Rotureau, N. Michel, M. Płoszajczak and B. R. Barrett, Phys. Rev. C $8 \mathbf{8}$ (2013) 044318.

3. A. Ekstrm, G. Baardsen, C. Forssn, G. Hagen, M. Hjorth-Jensen, G. R. Jansen, R. Machleidt and W. Nazarewicz et al., Phys. Rev. Lett. 110 (2013) 192502.

4. C. Forssén, Y. Kim, P. Maris, J. Rotureau, I. J. Shin and J. P. Vary, in preparation.

5. B. N. Parlett, The Symmetric Eigenvalue Problem, Prentice-Hall, 1980.

6. P. Sternberg, E. G. Ng, C. Yang, P. Maris, J. P. Vary, M. Sosonkina and H. V. Le, Accelerating configuration interaction calculations for nuclear structure, in Proc. of the 2008 ACM/IEEE conf. on Supercomputing, IEEE Press, Piscataway, NJ, p. 15:1 (2008).

7. P. Maris, M. Sosonkina, J. P. Vary, E. G. Ng and C. Yang, Proc. Comput. Sci. 1, 97 (2010).

8. H. M. Aktulga, C. Yang, E. G. Ng, P. Maris and J. P. Vary, Improving the scalability of symmetric iterative Eigensolver for multi-core platforms, Concurrency Computat.: Pract. Exper. DOI: 10.1002/cpe.3129 (2013, in press).

9. B. R. Barrett, P. Navratil and J. P. Vary, Prog. Part. Nucl. Phys. 69 (2013) 131.

10. A. M. Shirokov, A. I. Mazur, S. A. Zaytsev, J. P. Vary and T. A. Weber, Phys. Rev. C 70 (2004) 044005.

11. A. M. Shirokov, J. P. Vary, A. I. Mazur, S. A. Zaytsev and T. A. Weber, Phys. Lett. B 621 (2005) 96.

12. A. M. Shirokov, J. P. Vary, A. I. Mazur and T. A. Weber, Phys. Lett. B 644 (2007) 33.

13. R. Machleidt and D. R. Entem, Phys. Rept. 503 (2011) 1.

14. P. Maris, A. M. Shirokov and J. P. Vary, Phys. Rev. C 81 (2010) 021301

15. S. A. Coon, M. I. Avetian, M. K. G. Kruse, U. van Kolck, P. Maris and J. P. Vary, Phys. Rev. C 86 (2012) 054002.

16. R. J. Furnstahl, G. Hagen and T. Papenbrock, Phys. Rev. C 86 (2012) 031301

17. E. D. Jurgenson, P. Maris, R. J. Furnstahl, P. Navratil, W. E. Ormand and J. P. Vary, Phys. Rev. C 87 (2013) 054312.

18. C. Cockrell, J. P. Vary and P. Maris, Phys. Rev. C 86 (2012) 034325.

19. N. Michel, W. Nazarewicz, M. Płoszajczak and T. Vertse, J. Phys. G 36 (2009) 013101.

20. T. Berggren, Nucl. Phys. A 109 (1968) 265.

21. J. Rotureau, N. Michel, W. Nazarewicz, M. Płoszajczak and J. Dukelsky, Phys. Rev. C 79 (2009) 014304. 